\title{
Psychoseprävention jetzt in die klinische Versorgung einführen!
}

\author{
Introduce Prevention of Psychosis into Clinical Routine Now!
}

Autor

Institut

\section{Andreas Bechdolf}

Klinik für Psychiatrie, Psychotherapie und Psychosomatik, Vivantes Klinikum am Urban, Klinik für Psychiatrie und Psychotherapie, Universität zu Köln, Department of Youth Mental Health, University of Melbourne, Australia

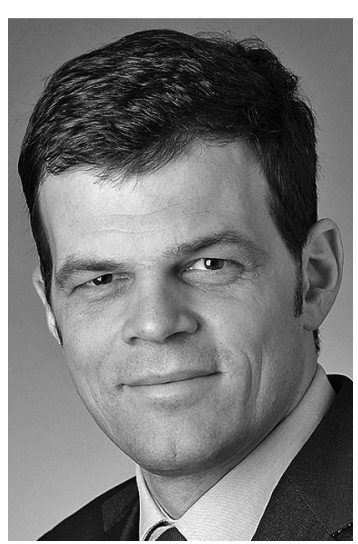

Prof. Dr. med. A. Bechdolf

\section{Bibliography \\ Dol http://dx.doi.org/ 10.1055/s-0032-1327393 \\ Online-Publikation: 0.0 . \\ Psychiat Prax 2013; 40: \\ 8-10 \\ (c) Georg Thieme Verlag KG Stuttgart · New York ISSN 0303-4259}

\section{Korrespondenzadresse Prof. Dr. med. A. Bechdolf, M. Sc. \\ Klinik für Psychiatrie, Psychotherapie und Psychosomatik, Vivantes Klinikum am Urban, Klinik für Psychiatrie und Psychotherapie, Universität zu Köln, Department of Youth Mental Health, University of Melbourne, Australia \\ Dieffenbachstraße 1 \\ 50924 Köln}

andreas.bechdolf@vivantes.de
Präventive Ansätze haben die Prognose einer Reihe von Volkserkrankungen wie z. B. arterieller Hypertonie oder Diabetes mellitus wesentlich verbessert. Damit der Einsatz eines präventiven Ansatzes zur Verhinderung des Auftretens einer Erkrankung in die klinische Regelversorgung gerechtfertigt ist, müssen 2 Voraussetzungen erfüllt sein: Erstens, muss die Identifikation eines Risikostadiums mit klinisch bedeutsamer Gefahr des Übergangs in das volle Erkrankungsbild möglich sein. Zweitens, müssen Interventionen vorliegen, die dieses Übergangsrisiko bei angemessen Nebenwirkungen in klinisch bedeutsamer Weise reduzieren.

Die schizophrene Störung gehört zu den 10 Erkrankungen, die mit den meisten in Behinderungen verlebten Jahren einhergehen (WHO, 2001). Sie zeigt in vielen Fällen einen chronischen Verlauf, kann für die Betroffenen und ihre Angehörigen mit schwerem und langjährigem Leid einhergehen und auf gesellschaftlicher Ebene erhebliche Kosten verursachen $[1,2]$. Vor diesem Hintergrund haben vor rund 20 Jahren erste Bemühungen um eine Prävention der schizophrenen Erkrankung begonnen. Bei der Prävention von Psychosen, wie sie derzeit betrieben wird, handelt es sich nicht um den Versuch einer Primärprävention universal in der Allgemeinbevölkerung oder selektiv bei noch gesunden Risikoträgern, sondern um eine indizierte Prävention bei Personen, die bereits klinisch symptomatisch sind und wegen der damit verbundenen Beschwerden und Einschränkungen aktiv Hilfe suchen. Mittlerweile wurden in den präventiv ausgerichteten Forschungsaktivitäten so viele Daten generiert, dass Psychoseprävention als wissenschaftlich begründet gelten kann und in die klinische Praxis eingeführt werden sollte.

Der aktuelle Wissenstand ist in den nächsten 3 Absätzen zusammengefasst.

\section{Läst sich eine psychotische Ersterkrankung vorhersagen? \\ $\nabla$}

Verschiedene Arbeitsgruppen haben Kriterien für ein klinisch erhöhtes Psychoserisiko entwickelt, welche im Wesentlichen unterschwellige psychotische Symptome [3] oder selbstwahrnehmbaren Denk- oder Wahrnehmungsstörungen (sog. Basissymptomen, [4]) umfassen.

Der erste Satz an Risikokriterien - die sog. ultrahigh risk“- (UHR-)Kriterien - beeinhalten 1. attenuierte (abgeschwächte) psychotische Symptome, 2. kurzzeitig vorhandene, spontan remittierende psychotische Symptome und 3. eine Kombination aus Risikofaktoren, wie eine schizophrene Erkrankung eines Angehörigen ersten Grades oder eine schizotype Persönlichkeitsstörung beim Betroffenen, und einem signifikanten Absinken des globalen Funktionsniveaus. Unter Basissymptome werden milde, meist subklinische, aber nichtsdestotrotz häufig einen starken Beschwerdedruck hervorrufende Selbstwahrnehmungen von Störungen des Antriebs, des Affekts, der Denk- und Sprachprozesse, der Wahrnehmung, der Propriozeption, der Motorik und der zentralvegetativen Funktionen verstanden.

Diese Risikokriterien sind reliabel zu erheben $[5,6]$ und wurden mittlerweile weltweit prospektiv auch an größeren Patientenkohorten evaluiert. In einer aktuellen Metaanalyse, in die Daten von etwa 2500 Betroffenen, welche diese Risikokriterien erfüllen, einbezogen wurden, wurden Übergangsraten in die psychotische Ersterkrankung von $18 \%$ nach 6 Monaten, $22 \%$ nach einem Jahr, $29 \%$ nach 2 Jahren und $36 \%$ nach 3 Jahren festgestellt [7]. 


\section{Können wir das Auftreten psychotischer Ersterkrankung verhindern? \\ $\nabla$}

Derzeit liegen 8 abgeschlossene randomisiert-kontrollierte Studien vor, welche low-dose Risperidon und kognitive Verhaltenstherapie [8], kognitive-verhaltenstherapeutische Intervention [9 -11], Olanzapin alleine [12] oder Omega-3-Fettsäuren [13] bei Personen mit erhöhtem Psychoserisiko evaluiert haben. Obwohl sich die letzte Cochrane-Analyse, welche sich auf 5 Studien bezog, vorsichtig äußerte, wegen der Heterogenität der Studien keine Metaanalyse durchführte und weitere Studien empfahl [14], liegen mittlerweile eine publiziert Metaanalyse [15] und 2 Metaanalysen unter Beteiligung des Autors dieses Beitrags vor, welche sich im Reviewverfahren befinden. Diese Metaanalysen haben zum Ergebnis, dass die Patienten der jeweiligen Experimentalbedingung ganz überwiegend einen günstigeren Symptomverlauf zeigen, eine bessere soziale Anpassung und weniger Übergänge in die Psychose aufweisen. Die präventiven Effekte, gemessen an der Anzahl der Patienten, die präventiv behandelt werden müssen um ein Ereignis zu verhindern (Number Needed to Treat - NNT), sind z.T. wesentlich stärker ausgeprägt als dies z.B. für die Prävention von Schlaganfällen durch antihypertensive Medikation bei Patienten mit arterieller Hypertonie bekannt ist (NNT zwischen 4 und 8 [16] vs. NNT 13). Erste Untersuchungen zeigen, dass Personen mit erhöhtem Psychoserisiko, die eine Frühintervention erhalten, im Vergleich zu psychotisch Ersterkrankten ohne Frühintervention im weiteren Verlauf weniger psychiatrische Behandlung in Anspruch nehmen, sodass die Frühintervention mittelfristig kostengünstiger als die Standardbehandlung ist [17].

\section{Was für Menschen erfüllen die Risikokriterien? $\nabla$}

Personen mit erhöhtem Psychoserisiko gehen oft durch eine lange Phase frustrierender Suche nach Unterstützung [18] und leiden unter klinischen Symptomen, die zu sozialer Behinderung und zu reduzierter subjektiver Lebensqualität führen [19]. Zwischen 60 und $80 \%$ der Betroffenen erfüllen zusätzlich zum Risikosyndrom Diagnosekriterien einer psychiatrischen Störung nach DSM-IV oder ICD-10, am häufigsten Depression und Angststörungen [20]. Darüber hinaus werden eine Reihe von schizophrenieähnlichen neurobiologischen Veränderungen bei Personen mit erhöhtem Psychoserisiko beschrieben (Übersicht bei [21]). Erste Studien zeigen, dass auch Risikopersonen, die keine psychotische Episode entwickeln, nach 2 Jahren massive soziale Behinderungen aufweisen und fast 50\% der Betroffenen noch weiterhin die Kriterien eines erhöhten Psychoserisikos erfüllen [22].

\section{Die Forschungsergebnisse rechtfertigen die Einfüh- rung von Psychoseprävention in die klinische Praxis $\nabla$}

Zusammenfassend lässt sich feststellen, dass die Bemühungen um eine Psychoseprävention zu prospektiv evaluierten Risikokriterien geführt hat, welche eine hilfesuchende Population identifiziert, die klinisch symptomatisch und funktionell beeinträchtigt ist. Diese Population zeigt ein Risiko relativ kurzfristig an einer psychotischen Störung zu erkranken, welches mehrere 100-fach über dem Erkrankungsrisiko der Normalbevölkerung liegt und deren Übergangszahlen in eine psychotische Erstmanifestation klinisch bedeutsam sind. Die bisher entwickelten Interventionen führen zu einer Verbesserung der zur Behandlung führenden Symptomatik und des Funktionsniveau und können das Auftreten psychotischer Episoden in klinisch bedeutsamer Weise zumindest verzögern, wenn nicht sogar mittelfristig verhindern. Auch unabhängig vom Übergangsrisiko zeigen Risikopersonen Symptome und Funktionseinschränkungen, die die allgemeinen Kriterien für psychiatrische Erkrankungen nach DSM-IV erfüllen und somit Behandlungsbedürftigkeit anzeigen [23].

Somit sind die beiden eingangs genannten Voraussetzungen zur Einführung präventiver Ansätze in die Regelversorgung erfüllt. Wie weit die internationale Akzeptanz der Ergebnisse der Präventionsforschung bei Psychosen mittlerweile geht, zeigt die Tatsache, dass ein Psychoserisikosyndrom in die Revision der aktuellen psychiatrischen Diagnosesysteme eingearbeitet werden soll. Unter dem Terminus „Attenuated Psychosis Syndrome“ (Abgeschwächtes Psychosesyndrom) wird es in die Forschungskriterien des neuen DSM-5 aufgenommen [24]. Bezüglich ICD-11, welche in der Entwicklung der Revision zeitlich zurückliegt, ist derzeit vorgeschlagen, ein abgeschwächtes Psychosesyndrom in die Z- oder R-Kategorie aufzunehmen [25]. Also, die Risikosymptome noch nicht einer Krankheitsentität zuzuordnen, aber es als ein Syndrom zu definieren, das Krankheitswert hat und Behandlung rechtfertigt.

\section{Wie sollte Psychoseprävention konzipierte sein? $\nabla$}

Auch wenn die bisherigen Studien zeigen konnten, dass die klinischen Symptome, die Funktionseinschränkung und die subjektive Belastung, wegen derer die Patienten Hilfe suchen, durch die Frühintervention signifikant gebessert werden und diese Effekte alleine die Frühintervention rechtfertigen könnten, sind bezüglich des präventiven Aspektes der Früherkennung und Frühintervention hohe ethische Maßstäbe anzulegen. Etwa 60\% aller Betroffenen werden zwar in Form von Symptomremission und Funktionsverbesserungen von präventiven Interventionen profitieren, jedoch im unbehandelten Verlauf keine Psychose entwickeln. Dementsprechend muss das jeweilige Aufklärungs- und Hilfsangebot durch eine verantwortungsbewusste Nutzen-Risiko-Abwägung zu rechtfertigen sein [26]. Alleine die Informationsvermittlung, einer Risikopopulation anzugehören, könnte potenziell angstauslösend und stigmatisierend wirken. Die Erfahrungen an den Früherkennungszentren zeigen jedoch, dass die empathische und individualisierte Informationsvermittlung in einem angemessenen Setting meist zur Entlastung bei den Betroffenen führt. Zu dieser Beobachtung liegen auch erste empirische Daten vor [27, 28]. Bezüglich der Art der Frühinterventionen sind akzeptable, wenig stigmatisierende und nebenwirkungsarme Interventionen wie Psychotherapie und Omega-3-Fettsäuren weniger risikobehaftet als antipsychotische Medikation (Übersicht zur ethischen Diskussion in [29]).

Folglich sollten Zentren, welche diagnostische und therapeutische Angebote für Risikopersonen vorhalten, einer Reihe von Anforderungen gerecht werden. Diese sind bei Häfner und Kollegen [30] detailliert beschrieben: Erstens sollten diese Früherkennungs- und Therapiezentren niederschwellig, nicht stigmatisierend und überwiegend ambulant konzipiert sein und darüber hinaus die spezifischen Bedürfnisse von jungen Erwachsenen und Jugendlichen reflektieren. Durch diese Konzeption soll die Zugänglichkeit des Angebots für die meist jungen Betroffenen und deren Angehörigen erleichtert werden. Zweitens sollten die Mit- 
arbeiter intensiv in der klinischen Erhebung eines erhöhten Psychoserisikos geschult sein und über die nötige Erfahrung in der klinischen Einschätzung verfügen. Drittens sollten die Mitarbeiter über ein konstruktives theoretisches Modell des Syndroms verfügen, innerhalb dessen sie den Patienten und ihren Angehörigen Informationen über das erhöhte Psychoserisiko und mögliche Bewältigungsversuche vermitteln können. Viertens sollten innerhalb der Zentren eine Reihe von Interventionsmöglichkeiten vorgehalten werden. Diese reichen von regelmäßigen Monitoringterminen, motivierender Gesprächsführung bei Substanzgebrauch, supportiver Therapie, kognitiver Verhaltenstherapie und Familientherapie über eine breite Palette von pharmakologischen Interventionen - von neuroprotektiven über antidepressive bis hin zu antipsychotischer Medikation. Dieses breit gefächerte Angebot ist nötig, um eine Intervention mit einem optimalen Nutzen-Risiko-Profil für die individuell Betroffenen auszuwählen.

\section{Die Kollegen in Australien zeigen wie es geht \\ $\nabla$}

Nachdem Australien, Großbritannien, die Niederlande und Dänemark bereits systematisch spezialisierte Behandlung für Ersterkrankte mit Psychosen in der klinischen Versorgung etabliert haben, hat die australische Regierung jetzt nachgelegt [31]. Sie hat entschieden 600 Million \$ in den nationalen Aufbau von niederschwelligen, spezialisierten Zentren für Jugendliche und junge Erwachsene zu investieren, die insbesondere unter Risikosymptomen für psychotische Störungen leiden. Die Einführung von abgeschwächten psychotischen Symptomen in die Z- oder R-Kategorie von ICD-11 könnte auch in Deutschland Startpunkt für Verhandlungen mit Kostenträgern sein, die Gründung von spezialisierten Zentren für Patienten mit Risikosymptomen zu fördern. Auf diese Art und Weise könnte ein Paradigmenwechsel von der Therapie zur Prävention der schizophrenen Erkrankung eingeleitet werden. Dieser Paradigmenwechsel bietet die einzigartige Chance für viele Betroffene, einen besseren Krankheitsverlauf zu erreichen, Behinderung zu reduzieren und das Leid bei Betroffenen und Angehörigen zu minimieren sowie gesellschaftliche Kosten zu reduzieren. Diese Chance sollten wir jetzt ergreifen.

\section{Literatur}

1 van Os J, Kapur S. Schizophrenia. Lancet 2009; 374: 635-645

2 Konnopka A, Klingberg S, Wittorf A et al. The cost of schizophrenia in Germany: a systematic review of the literature. Psychiat Prax 2009; 36: $211-218$

3 Yung AR, Phillips LJ, Yuen HP et al. Psychosis prediction: 12-month follow up of a high-risk ("prodromal") group. Schizophr Res 2003; 60: $21-32$

4 Klosterkotter J, Hellmich M, Steinmeyer EM et al. Diagnosing schizophrenia in the initial prodromal phase. Arch Gen Psychiatry 2001; 58: $158-164$

5 Miller TJ, McGlashan TH, Rosen JL et al. Prospective diagnosis of the initial prodrome for schizophrenia based on the Structured Interview for Prodromal Syndromes: preliminary evidence of interrater reliability and predictive validity. Am J Psychiatry 2002; 159: 863-865

6 Yung AR, Yuen HP, McGorry PD et al. Mapping the onset of psychosis: the Comprehensive Assessment of At-Risk Mental States. Aust N Z J Psychiatry 2005; 39: 964-971
7 Fusar-Poli P, Bonoldi I, Yung AR et al. Predicting psychosis: meta-analysis of transition outcomes in individuals at high clinical risk. Arch Gen Psychiatry 2012; 69: 220-229

8 McGorry PD, Yung AR, Phillips LJ et al. Randomized controlled trial of interventions designed to reduce the risk of progression to first-episode psychosis in a clinical sample with subthreshold symptoms. Arch Gen Psychiatry 2002; 59: 921 - 928

9 Morrison AP, French $P$, Walford $L$ et al. Cognitive therapy for the prevention of psychosis in people at ultra-high risk: randomised controlled trial. Br J Psychiatry 2004; 185: 291 - 297

10 Addington J, Epstein I, Liu L et al. A randomized controlled trial of cognitive behavioral therapy for individuals at clinical high risk of psychosis. Schizophr Res 2011; 125: 54-61

11 Bechdolf A, Wagner M, Ruhrmann $S$ et al. Preventing progression to first-episode psychosis in early initial prodromal states. Br J Psychiatry 2012; 200: 22-29

12 McGlashan TH, Zipursky RB, Perkins D et al. Randomized, double-blind trial of olanzapine versus placebo in patients prodromally symptomatic for psychosis. Am J Psychiatry 2006; 163: 790 - 799

13 Amminger GP, Schafer MR, Papageorgiou $K$ et al. Long-chain omega-3 fatty acids for indicated prevention of psychotic disorders: a randomized, placebo-controlled trial. Arch Gen Psychiatry 2010; 67: 146-154

14 Marshall M, Rathbone J. Early intervention for psychosis. Cochrane Database Syst Rev 2011: DOI: 10.1002/14651858.CD004718.pub3 CD004718

15 Preti A, Cella M. Randomized-controlled trials in people at ultra high risk of psychosis: a review of treatment effectiveness. Schizophr Res 2010; 123: $30-36$

16 Muller H, Wiessmann T, Bechdolf A. Interventionen bei Personen mit erhöhtem Psychoserisiko: Eine aktuelle Übersicht über randomisiert kontrollierte Studien. Fortschr Neurol Psychiatr 2012; 80: 570-579

17 Phillips LJ, Cotton S, Mihalopoulos C et al. Cost implications of specific and non-specific treatment for young persons at ultra high risk of developing a first episode of psychosis. Early Interv Psychiatry 2009; 3 : $28-34$

18 Kohn D, Pukrop R, Niedersteberg A et al. Pathways to care: help-seeking behavior in first-episode psychosis. Fortschr Neurol Psychiatr 2004; 72: $635-642$

19 Bechdolf A, Pukrop R, Kohn D et al. Subjective quality of life in subjects at risk for a first episode of psychosis: a comparison with first episode schizophrenia patients and healthy controls. Schizophr Res 2005; 79: $137-143$

20 Svirskis T, Korkeila J, Heinimaa $M$ et al. Axis-I disorders and vulnerability to psychosis. Schizophr Res 2005; 75: 439-446

21 Correll CU, Hauser M, Auther AM et al. Research in people with psychosis risk syndrome: a review of the current evidence and future directions. J Child Psychol Psychiatry 2010; 51: 390 - 431

22 Addington J, Cornblatt BA, Cadenhead KS et al. At clinical high risk for psychosis: outcome for nonconverters. Am J Psychiatry 2011; 168: $800-805$

23 American Psychiatric Association. Diagnostic and Statistical Manual of Mental Disorders. DSM-IV. 4th Edition. Arlington: American Psychiatric Association; 2000

24 American Psychiatric Association. DSM-5 Development. 2011: http:// www.dsm5.org/Pages/Default.aspx

25 Gaebel W, Reed GM. Status of Psychotic Disorders in ICD-11. Schizophr Bull 2012; 38: 895 - 898

26 Bechdolf A, Peukert R. Psychosis prevention: expectations met. Psychiat Prax 2011; 38: 58-60

27 Hauser M, Lautenschlager M, Gudlowski Y et al. Psychoeducation with patients at-risk for schizophrenia - an exploratory pilot study. Patient Educ Couns 2009; 76: 138-142

28 Zarafonitis SWM, Pützfeld V, Berning J et al. Psychoedukation bei Personen mit erhöhtem Psychoserisiko. Psychotherapeut 2012; 57: 326 334

29 DeLisi L. Schizophrenia Research 2001; 51

30 Häfner H, Bechdolf A, Klosterkötter J et al. Psychosen - Früherkennung und Frühintervention. Stuttgart: Schattauer; 2012

31 Australia Medical Association. A Ten Year Roadmap for National Mental Health. 2012: https://ama.com.au/submission-draft-ten-year-road map-national-mental-health-reform 